\title{
Energy Efficient Wireless Sensor Networks using Co- operative MIMO: A Technical Review
}

\author{
Vibhav Kumar Sachan \\ Department of Electronics \& \\ Communication Engineering \\ KIET Group of Institutions, \\ Ghaziabad, U.P, India
}

\author{
Richa Maheshwari \\ Department of Electronics \& \\ Communication Engineering \\ KIET Group of Institutions, \\ Ghaziabad, U.P, India
}

\author{
Syed Akhtar Imam \\ Department of Electronics \& \\ Communication \\ EngineeringJamia Millia \\ Islamia, New Delhi, India
}

\begin{abstract}
This paper considers the wireless sensor networks and highlights its applications in present time and mainly focuses on the two main techniques for saving maximum energy in transmitting information in WSN and these are multi-input multi-output (MIMO) and cooperative MIMO (CMIMO). Under this paper there is a comparison of energy efficiency in MIMO and single-input single-output (SISO) system for both transmission energy and circuit energy. Then there will be the discussion of CMIMO advantages, its energy model, properties, comparison with the equivalent technique (DCA) and the most important strategy used under CMIMO system is node sleep strategy and its significance in front of non-node sleep strategy will be discuss in this paper.
\end{abstract}

\section{Keywords}

Wireless sensor networks, MIMO system, CMIMO technology, energy efficiency, node sleep strategy.

\section{INTRODUCTION}

Wireless sensor network is a very interesting area to focus on because of its real time applications as: Habitat monitoring, object tracking, military systems and industrial areas. Wireless sensor network (WSN) basically composed of hundreds or more number of nodes and having wide range of communication. In WSN, different sensor nodes can communicate with each other wirelessly. In these systems communication can be very efficient using different transmission schemes, as MIMO, CMIMO etc. In these networks, sensor (an electronic device) used as an interface between its source and destination, through which sensor nodes can communicate with one another to form a network. Sensor nodes are consists of different no. of elements as ADC, DAC, processor and others just on a single chip powered by batteries mostly, whose replacement is very difficult and expensive. Hence energy minimization is a main design criterion in wireless sensor network for which different diversity schemes are used. Most common is cooperative MIMO technology which yields tremendous increase in energy saving.
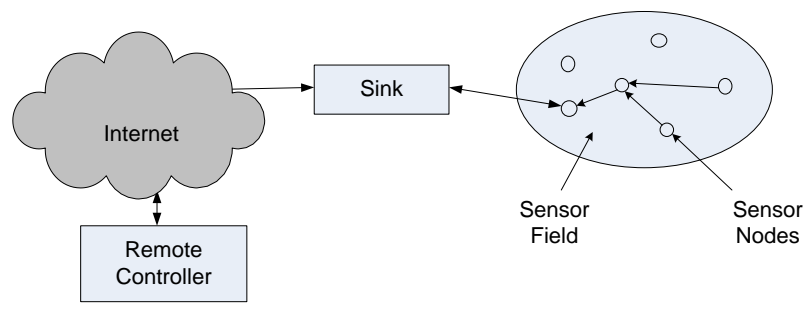

User

Fig.1: Wireless Sensor Network Model
Basically energy saving methods focus on two subsystems: the networking subsystem (i.e., for the designing of networking protocols as well as for the operation of each single node energy management is concerned), and the sensing subsystem (i.e., to decrease the frequency of energy expensive sample, different techniques are used). Wireless sensor network can be applicable in many fields as: in military target tracking and surveillance, a WSN can help in intrusion detection and identification. In natural disasters sensor nodes can sense and detect the environment to forecast disasters before their occurrence. In biological medical applications, in monitoring a patient's health, surgical implants of sensors can be used. WSN is having its own design and resource limitation as low bandwidth, limited processing, short range communication, storage capacity in each node.

\section{ENERGY CONSUMPTION AND ITS OPTIMIZATION IN DIFFERENT COMPONENTS OF SENSOR NODES}

Generally, sensor nodes are battery powered devices therefore the main concern is to reduce the energy consumption so that the network lifetime can be increased. Under this topic first, energy consumption in different components of sensor node and different energy saving methods will be described. Sensor nodes are basically consists of RF transceiver, A/D and D/A convertors, baseband processors and other applications interface integrated into a single device.

The sensor node is mainly comprises of three units.

1. Sensing unit: It sense the information from neighboring atmosphere about temperature change, pressure change, information about unwanted data from enemy etc. For this it consumes energy from battery or other source of energy.

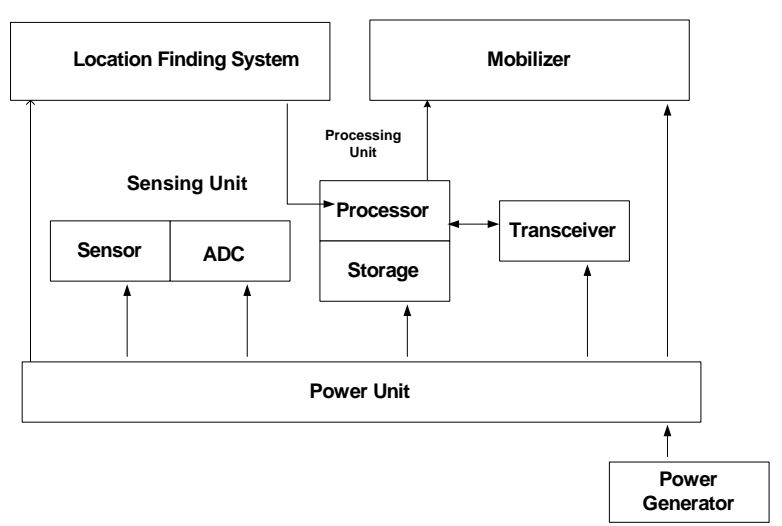

Fig.2: Components of sensor nodes 
2. Data Processing unit: Sensing unit transmit the collected data to the processor, which then process data according to the destination and put processed data to the communication unit.

3. Communication unit: This is the most important unit which consumes much higher energy than above two. Its function leads to the completion of information transfer in wireless sensor network.

The power consumption mainly depends upon particular node, as the power characteristics of mote class node are completely different from stargaze node. Depending upon the above power consumptions in different unit, several approaches have to be used, to reduce power consumption in wireless sensor network.

\subsection{Energy Saving Methods in wireless sensor network}

There are three main enabling techniques namely, duty cycling, data driven approaches and mobility [2].

\subsubsection{Duty Cycling Approaches}

This approach mainly focused on "networking subsystem". Duty cycling is defined as fraction of time nodes are active during their lifetime. Basically this approach is based on Topology control and power management.

\subsubsection{Data Driven Approaches:}

This energy efficient data acquisition schemes are mainly focused on reducing energy spent by "sensing subsystem". These approaches are planned to reduce the amount of sampled data by keeping the sensing accuracy which can be acceptable for applications. This approach is based on Data Reduction and Energy-Efficient Data Acquisition.

\subsubsection{Mobility Based Approach:}

Mobility is helpful in reducing energy consumption as; packets coming from sensor node negotiate the network towards sink by following multi-hop path .When the sink is static; a few paths can be loaded high than others based on network topology and packet generation rates at sources. Mobility based schemes can be classified depending on nature of mobile element as: Mobile Sink (MS) and Mobile Relay (MR).

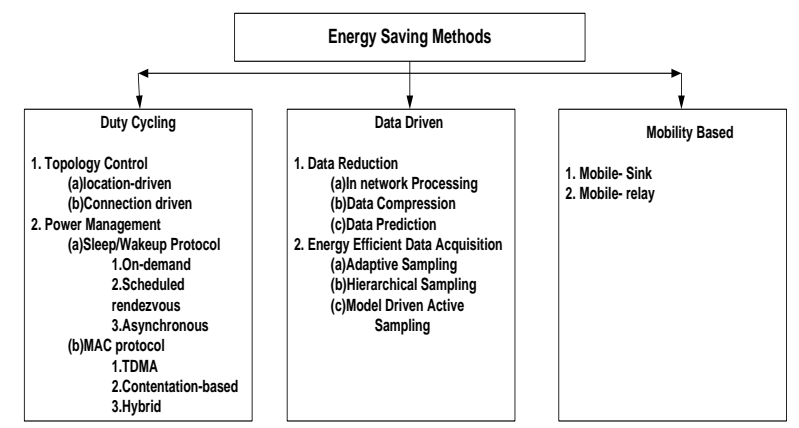

Fig.3: Different conservation schemes in WSN

\section{ENERGY EFFICIENT WIRELESS SENSOR NETWORKS USING MIMO SCHEME}

The huge numbers of nodes in WSN are powered by the batteries which are difficult or even impossible to replace or recharge in most cases. Hence, for energy efficient transmission, minimizing energy consumption becomes extremely important in such energy constrained networks.
So, we use "MIMO System" based on antenna arrays provides effective way for energy saving.

\subsection{Introduction to MIMO}

MIMO stands for multi-input multi-output technique which has many advantages over normal SISO (single-input singleoutput). It's a multi antenna system, due to its great capability of increasing channel capacity in fading channels it has become an intensive topic for new researchers in recent years. Utilizing spatial diversity, MIMO can significantly reduce the required transmitting power under fixed performance requirement such as throughput and packet error rate (PER). Now for showing energy consumption we just consider general link of communication which can be multi-input multi-output (MIMO), multi input single output (MISO), single input multi output (SIMO) or single-input single-output (SISO).

With the help of the block diagrams below (Fig.4 and Fig.5) the energy consumption can be shown as

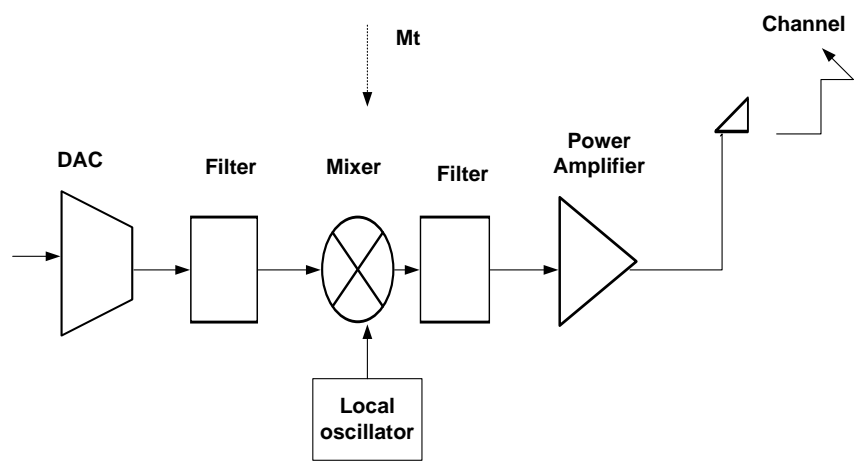

Fig.4: Transmitter circuit blocks (analog)

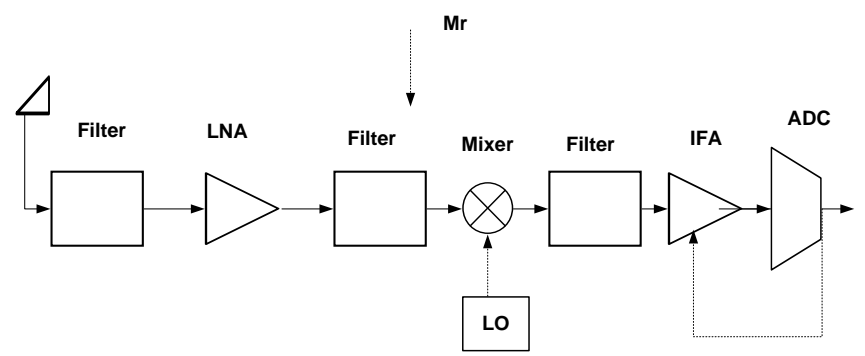

Fig.5: Receiver circuit blocks (analog)

The above block diagram shows the circuit arrangement of the system, where $M_{t}$ and $M_{r}$ are the number of antennas at transmitter and receiver respectively. $M_{t}$ and $M_{r}$ are one for SISO and more for MIMO.

\subsection{Energy model of MIMO systems}

The total average power consumption along the signal path can be divided into two main components: the consumption of power in all the power amplifiers and the consumption of power in the other circuit blocks. The power in power amplifiers is dependent on the transmit power $P_{\text {out }}$ which can be calculated according link budget relationship as:

$P_{\text {out }}=\bar{E}_{b} R_{b} \times \frac{(4 \pi d)^{2}}{G_{t} G_{r} \lambda^{2}} M_{l} N_{f}$

Where, $\bar{E}_{b}$ is required energy per bit at the receiver. $R_{b}$ is the bit rate, $d$ is the transmission distance, $G_{t}$ and $G_{r}$ are transmitter and receiver antenna gain respectively. $\lambda$ Is carrier wavelength, $M_{l}$ is link margin and $N_{f}$ is receiver noise figure which is 
defined $\operatorname{asN}_{\mathrm{f}}=\left(\mathrm{N}_{\mathrm{r}} / \mathrm{N}_{\mathrm{o}}\right)$, where $\mathrm{N}_{\mathrm{o}} / 2=-174 \mathrm{dBm} / \mathrm{Hz}$ and $\mathrm{N}_{\mathrm{r}}$ is the PSD of total effective noise at the receiver input.

The approximate value of power consume by power amplifiers is:

$\mathrm{P}_{\mathrm{PA}}=(1+\alpha) \mathrm{P}_{\text {out }}$

Where, $\alpha=(\xi / \eta)-1$ with $\eta$ is the drain efficiency[6] of RF power amplifier and $\xi$ is the peak to average ratio (PAR) which is dependent on the modulation scheme and respective constellation size[7]. The second important term is $P_{c}$ which is total circuit power and can be defined as:

$$
\begin{aligned}
\mathrm{P}_{\mathrm{c}}=\mathrm{M}_{\mathrm{t}}\left(\mathrm{P}_{\mathrm{DAC}}+\mathrm{P}_{\mathrm{mix}}+\mathrm{P}_{\text {filt }}\right)+2 \mathrm{P}_{\text {syn }} \\
+\mathrm{M}_{\mathrm{r}}\left(\mathrm{P}_{\mathrm{LNA}}+\mathrm{P}_{\mathrm{mix}}+\mathrm{P}_{\mathrm{IFA}}+\mathrm{P}_{\text {filt }}+\mathrm{P}_{\mathrm{ADC}}\right)
\end{aligned}
$$

Where, $\mathrm{P}_{\mathrm{DAC}}, \mathrm{P}_{\text {mix }}, \mathrm{P}_{\text {filt }}, \mathrm{P}_{\text {sync }}, \mathrm{P}_{\mathrm{LNA}}, \mathrm{P}_{\mathrm{IFA}}, \mathrm{P}_{\mathrm{ADC}}$ are the values of power consumed in DAC, the mixer, low noise amplifier (LNA), the intermediate frequency amplifier (IFA), active filters at transmitter side, the active filter at receiver side, the ADC and frequency synthesizer respectively. To find the estimated values of $\mathrm{P}_{\mathrm{DAC}}, \mathrm{P}_{\mathrm{IFA}}, \mathrm{P}_{\mathrm{ADC}}$, we use the model given in [7].

Then, finally the total energy consumption per bit for fixed-rate system is given by:

$\mathrm{E}_{\mathrm{bt}}=\left(\mathrm{P}_{\mathrm{PA}}+\mathrm{P}_{\mathrm{c}}\right) / \mathrm{R}_{\mathrm{b}}$

In the MIMO systems Alamouti schemes are used for diversity. For two transmitting antennas the Alamouti code [8] uses two different symbols $s_{1}$ and $s_{2}$ transmitted simultaneously from both antenna 1 and 2 respectively. Alamouti code for more than two antennas is discussed in [5]. It has been shown in [5] that for Rayleigh-fading channels MIMO system based on Alamouti schemes will have lower probability of error than SISO system. So under the same BER and throughput requirement, MIMO will require less transmission energy than SISO systems. But in the case of circuit power consumption it is not clear that which system is more efficient as the circuit of MIMO system consumes huge amount of power. Total energy defined above is its general expression, MIMO systems are generally classified in two major fields in which its total energy consumption is defined by a different formula as:

\subsubsection{Fixed rate system with BPSK modulation:}

In this part it is assumed that a channel gain between each transmitter and receiver antenna is a scalar i.e., flat Rayleighfading channel. So MIMO channel's fading factors can be represented as a scalar matrix and additionally path loss is modeled as power falloff which is proportional to the square of distance. The signal is attenuated by fading matrix $\mathrm{H}$, for square-law path loss in which each entry is zero-mean circulant symmetric complex Gaussian random variable (ZMCSCG) with unit variance [5]. The values of power consumption in various circuit blocks can be taken from [9]-[12]. Under this scheme there are two sub-parts which describe energy consumption in MISO and MIMO systems that use Alamouti schemes with BPSK modulation and their energy efficiency comparison with SISO system.

(1) Alamouti $2 \times 1$ : In this scheme $\mathrm{H}=\left[\mathrm{h}_{1} \mathrm{~h}_{2}\right]$ and the reference SISO system is consider as a special case of MISO system with $\mathrm{H}=\left[\mathrm{h}_{1}\right]$.

The received SNR is defined as:
$\gamma_{\mathrm{b}}=\frac{\|\mathrm{H}\|_{\mathrm{F}}^{2}}{\mathrm{M}_{\mathrm{t}}} \frac{\overline{\mathrm{E}_{\mathrm{b}}}}{\mathrm{N}_{0}}, \quad \mathrm{M}_{\mathrm{t}}=1,2$

The average BER is given by [5]

$\overline{\mathrm{P}_{\mathrm{b}}}=\epsilon_{\mathrm{H}}\left\{\mathrm{Q}\left(\sqrt{2 \gamma_{\mathrm{b}}}\right)\right\}$.

And according to Chernoff bound [5]

$\overline{\mathrm{P}_{\mathrm{b}}} \leq\left(\frac{\overline{\mathrm{E}_{\mathrm{b}}}}{\mathrm{M}_{\mathrm{t}} \mathrm{N}_{\mathrm{o}}}\right)^{-\mathrm{M}_{\mathrm{t}}}$

Now we can derive an upper bound for the required energy per bit as:

$\overline{\mathrm{E}_{\mathrm{b}}} \leq \frac{\mathrm{M}_{\mathrm{t}} \mathrm{N}_{\mathrm{o}}}{\overline{\mathrm{P}}_{\mathrm{b}}} \mathrm{M}_{\mathrm{t}}$

Then total energy consumption is given by:

$E_{b t}=(1+\alpha) \frac{M_{t} N_{o}}{\overline{P_{b}} 1 / M_{t}} \times \frac{(4 \pi d)^{2}}{G_{t} G_{r} \lambda^{2}} M_{l} N_{f}+P_{c} / R_{b}$

(2) Alamouti $2 \times 2$ : Now considering the case of $2 \times 2$ MIMO systems based on Alamouti code where channel matrix is given by $\mathrm{H}=\left[\begin{array}{l}\mathrm{h}_{11} \mathrm{~h}_{21} \\ \mathrm{~h}_{12} \mathrm{~h}_{22}\end{array}\right]$. In this MIMO system the diversity order of 4 and array gain of 2 is achieved and indicates that in this system, less transmission energy is consumed in comparison to the $2 \times 1$ MISO system under same performance requirement [10]. But during the comparison in the circuit energy consumption, MIMO adds more circuit energy consumption than in MISO.

\subsubsection{Variable rate systems}

As in the fixed rate assumption where the bit rate is $10 \mathrm{~kb} / \mathrm{s}$ and the modulation scheme is BPSK, there has been a comparison of the performance of the two systems (MISO and MIMO) with SISO [10] and it is shown that SISO can beat MIMO in terms of energy efficiency for short-range applications. However, for a data network the traffic is usually bursty and the packet-by-packet communication is going on. Suppose $\mathrm{L}$ bits in the transmitter buffer and we have a time limit $\mathrm{T}$ to finish the transmission of these $\mathrm{L}$ bits. The best and optimal strategy to reduce the total energy consumption is to operate on a multimode basis, which provides a huge saving in energy amount when sleep mode is deployed. The time spends by transceiver is Ton $\leq \mathrm{T}$ for transmission and reception of these bits, where Ton is the parameter to optimize corresponds to an optimal constellation size $\mathrm{b}$ (bits per symbol) and we have $\mathrm{b}=$ (L/BTon), where B is the modulation bandwidth for the MIMO systems [10].In a brief way we can define a new total energy formula given in [10] as:

$E_{b t}=(1+\alpha) \frac{M_{t} N_{o}}{\bar{P}_{b}^{1 / M_{t}}} \times \frac{(4 \pi d)^{2}}{G_{t} G_{r} \lambda^{2}} M_{l} N_{f}+\mathrm{P}_{\mathrm{c}} \mathrm{T}_{\mathrm{on}} / \mathrm{R}_{\mathrm{b}}$

Where, the above parameters are clearly and deeply defined in [10]

Several advantages of MIMO over SISO systems has been shown but after all the complex circuitry and high energy consumption in it MIMO cannot be easily used in every application. To combat the disadvantages of MIMO the CMIMO has been introduced. CMIMO will be described in the next section

\section{CO-OPERATIVE MIMO}

As in WSN, the nodes usually have a platform for small operation and are not practical to equip with multiple antennas due to size limitation. So, we use co-operative multi-input 
multi-output technique (CMIMO) technique which is based on clustered random wireless network just like the LEACH protocol [3]. There are also some another kind of cooperative communication in clustered wireless sensor network [7].

In CMIMO multiple nodes placed adjacently with single antenna form a virtual antenna array [6] to achieve spatial diversity. This technique is completely suitable for clustered WSN. MIMO links can improve energy efficiency in WSN. Though a node is equipped with only single antenna it is possible to make a virtual MIMO link by large number of sensor nodes. Such grouping can be formed using clustering.

\subsection{Distributed MIMO adaptive energy efficient clustering/ routing scheme (CMIMO)}

The objective of this scheme [4] is reducing the energy consumption in multihop WSN. It employs a dynamic clustering approach with two cluster heads per cluster: a master cluster head(MCH) and a slave cluster head( $\mathrm{SCH})$. The two cluster heads operate as a cooperative MIMO node for inter cluster communication.

The operation of CMIMO has three main phases:

\subsubsection{Cluster formation}

It is done in a distributed way, and results in atmost two cluster heads per cluster, where $\mathrm{MCH}$ is mandatory whereas $\mathrm{SCH}$ may or may not be present.

\subsubsection{Intra-cluster communication:}

During this communication, for aggregating the information from other sensor nodes and exchanging this information with $\mathrm{SCH}$, selected $\mathrm{MCH}$ is responsible. Therefore, the two clustered heads operate as a cooperative MIMO node (if required).

\subsubsection{Inter-cluster commiunication}

when the information is available at the cluster heads of source micro-cluster, inter cluster communication is carried out by forwarding or exchanging data with other micro-cluster or directly with sink.

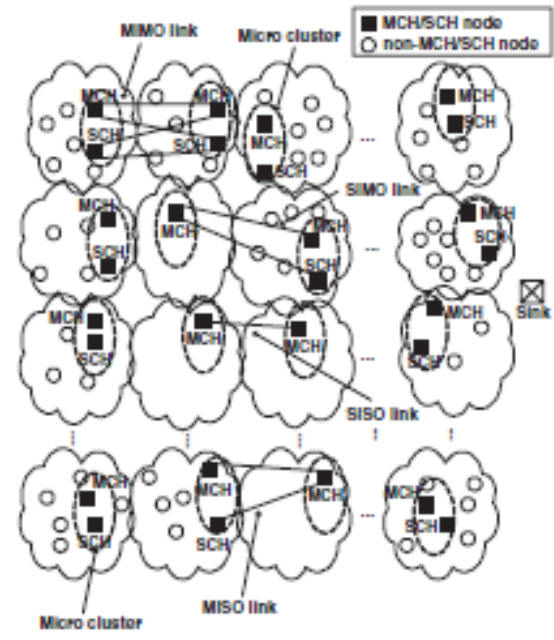

Fig.6: Example topology of a clustered WSN with CMIMO

\subsection{Properties of Co-operative MIMO}

Co-operatve MIMO has features as follows:
1. This is completely Distributed and that is the reason every node in WSN is completely independent and take its decisions based on local information.

2. At the end of clustering process, a node is either a $\mathrm{CH}$ (master or slave) or a non- $\mathrm{CH}$ node that belongs to a cluster or clustering process is guaranteed to terminate. It can be proven as the node with highest remaining energy in its neighbor is selected as a $\mathrm{MCH}$. This $\mathrm{MCH}$ then selects an SCH according to neighbor list criterion. Each non- $\mathrm{CH}$ node selects one of the MCHs to join.

3. An SCH can't belong to more then one cluster. Hence, invited $\mathrm{SCH}$ responds to only one of the received requests from $\mathrm{MCHs}$.

4. Probability that two nodes within eachn other's cluster range are both $\mathrm{MCHs}$ is zero, means $\mathrm{MCHs}$ are completely distributed.

\subsection{Designing Issues in CMIMO}

It include: Listening cost, Synchronization, Reclustering and MAC.

\subsubsection{Listening Cost}

There are some approaches that can be used by CMIMO to reduce the listening cost of active nodes. To ensure intercluster routing, $\mathrm{MCH}$ and $\mathrm{SCHs}$ should always be available. Whereas, non- $\mathrm{CH}$ nodes after sending their data to their $\mathrm{MCH}$ can be put to sleep. To reduce their energy consumption, $\mathrm{CHs}$ can also follow some duty cycle as follows: When a packet is intended to a $\mathrm{CH}$, the $\mathrm{CH}$ should be awake for a duration of time, which is needed to receive the incoming data. This, however requires coordination between the $\mathrm{CH}$ and its non- $\mathrm{CH}$ nodes. Other general approaches can also be used for coordinating communication. Such as S-MAC, T-MAC and TRAMA.

\subsubsection{Synchronisation:}

CMIMO design can be either in synchronous or asynchronous modes. Some approaches were proposed to solve the synchonization issue. One of them was using Reference Broadcast Synchronisation technique which can be adapt as:

An $\mathrm{MCH}$ asks one of its cluster nodes (except $\mathrm{SCH}$ ) to send RBS beacons. $\mathrm{MCH}$ and $\mathrm{SCH}$ start sending data simultaneously to $\mathrm{CHs}$ of receiving cluster after exchanging the RBS beacons and another scheme which has been used was transmission delay and channel estimation. The main drawbacks of these approaches are large no. of generated messages, the long elapsed time in overall synchronization and they do not consider the energy requirement of sensor nodes.

To solve these drawbacks, there is a solution to synchronisation issue according to which, select a node in a network to act as a beacon cluster head(BCH). Such a node sends beacon to its neighboring MCHs, So that they adjust the starting time of frames.

\subsubsection{Reclustering:}

The basic idea of reclustering is that once the remaining energy for any $\mathrm{MCH}$ falls below a threshold(e.g., $30 \%$ of its initial value), then this $\mathrm{MCH}$ sends a "reclustering" message to its neighboring $\mathrm{MCH}$ at power level $\left(P_{\text {inter }}\right)$. The purpose of this reclustering message is to inform other nodes that the present $\mathrm{MCH}$ requests reclustering .

The "hello" messages in the neighborhood discovery process in the "cluster formation" phase, is similar to the reclustering 
message. The only difference between both is that reclustering messages are sent at higher power level $\left(\mathrm{P}_{\text {inter }}\right)$.

As a result the neighboring $\mathrm{MCHs}$ that listen to these messages respond as in neighborhood discovery process. The rationale behind restricting reclustering request to $\mathrm{MCHs}$ is that, $\mathrm{MCHs}$ are the ones who deplete their batteries first (before SCHs and non- $\mathrm{CH}$ nodes), as it is responsible for aggregating data sending it to $\mathrm{SCH}$ and forward it to $\mathrm{CHs}$ in neighboring cluster.

\subsubsection{Medium Access Control:}

The two issues related to MAC layer are discussed as:

\subsubsection{Strategy of reliable communication (taking} packet losses into account).

By using ACKs at MAC layer for all network transmissions reliable communication can be established. These include:

- Transmissions for neighborhood discovery (where ACKs are represented by hello messages for previously heard hello messages)

- Between a non-CH and its $\mathrm{MCH}$ (e.g., "membership request messages" is acknowledged by "membership list messages").

- Between an $\mathrm{SCH}$ and its $\mathrm{MCH}$ (e.g., an "SCH acceptance message" is acknowledged by an "SCH confirmation message").

- Between an SCH/MCH in a transmitting cluster and an $\mathrm{SCH} / \mathrm{MCH}$ in a receiving cluster (e.g., MCH's and SCH's CPREQ packets are acknowledged by MCH's CPRES)

Additionally, CMIMO retransmits the non-acknowledged packets (limited to specific number of retransmissions) to increase the chances of correct reception of these packets.

\subsubsection{Selection of transmission range for intra-} and inter-cluster communication

For this Code Division Multiple Access (CDMA) Technique [5] is used, where for intra- and inter- cluster transmission different coding schemes are used. However, hardware implications of such technique may make it infeasible. Therefore another approach is proposed, in which transmission range for inter-cluster communication $\left(\mathrm{R}_{\mathrm{t}}\right)$ is selected a priori. To satisfy the connectivity condition, intra-cluster range is then selected. This approach has less complexity then CDMA approach.

\subsection{Performance evaluation of CMIMO}

In this section the performance of CMIMO will be evaluated and also the comparison between CMIMO with DCA will be discussed, which is one of the popular clustering schemes. The reason for opting DCA to compare is that, DCA resembles CMIMO in the criterion used to select CHs. Primary objective of this comparison is to demonstrate the benefits of using CMIMO over single antenna system (DCA). CMIMO adapts transmission modes and transmission power on per packet basis. Whereas all transmissions in DCA takes place using the SISO mode, where each cluster has only one $\mathrm{CH}$. This section includes several points as energy consumption, transmission modes and number of hops. So we start the comparison with energy consumption.

\subsubsection{Energy consumption}

This part contains two classifications for energy consumption in CMIMO and DCA as:

\subsubsection{Energy consumption versus inter cluster range}

Impact of inter-cluster range (the maximum range for direct communications between two clusters) on the total energy consumption for CMIMO and DCA is shown via graph in [4]. Results illustrates that total energy consumption increases with inter-cluster range. CMIMO is better in performance from DCA. Efficiency of CMIMO becomes more efficient as the inter-cluster range increases. The reason behind is that increasing inter-cluster range forces clusters that are far away from each other to communicate using multi-antenna modes. In this case transmission energy dominates circuit energy; making MIMO/MISO/SIMO more efficient than SISO mode. Thus CMIMO system is efficient under large inter-cluster ranges.

\subsubsection{Energy consumption versus Network Size}

The variation in the transmission distance leads to different ratios between transmission and circuit energies specifically transmission energy increases with distance while circuit energy remains same. Graph in [4] shows the energy consumption versus network size length i.e., length of square. We conclude from the figure that CMIMO has better energy performance than DCA, especially for larger areas.

\subsubsection{Transmission Modes}

Inter-cluster range basically affects how often various transmission modes are used. As we know that the maximum range for direct (1-hop) communications between two clusters is represented by inter-cluster range.

[4] Shows the frequent use of MIMO at higher values of intercluster ranges. This concludes from the fact that distances between two cluster heads of different clusters

become larger and thus the transmission energy dominates circuit energy. Then MIMO is used for the communication between the two cluster heads and as inter-cluster range increases the use of SISO decreases.

\subsubsection{Number of Hops}

In this section the impact of inter-cluster range and network area on the number of hops used for inter-cluster communications to the sink is studied. It can be shown with the help of a bar graph [4] of number of hops needed for routing under different inter-cluster ranges. When inter-cluster range is large (around $1 \mathrm{~km}$ ) then number of hops needed is less. While large number of hops exist when the inter-cluster range is small.

\section{NODE SLEEP STRATEGY IN WIRELESS SENSOR NETWORKS 5.1 Introduction to Node Sleep Strategy}

Newly proposed node sleep strategy for energy efficient cooperative transmission when the circuit energy consumption is also taken into consideration in random clustered WSN. The main aim of this proposed strategy is to minimize total energy consumption by selecting inter-cluster transmission energy consumption and the number of sensor nodes in activation. The relationship among the sensor node active rate and overall energy consumption is described in [1]. This strategy shows how we save energy in random wireless sensor network having CMIMO technique where overall energy consumption is minimized by coordinating inter-cluster transmitting energy consumption and the number of sensor nodes in activation. Therefore, 
- $\quad$ First, we study CMIMO energy consumption and divide it into intra-cluster energy consumption and inter-cluster energy consumption.

- We analyze the tradeoff between overall energy consumption and nodes active rate.

Further we discuss how these parameters are affected by nodes density, inter-cluster transmitting distance, cluster radius and intra-cluster energy consumption and finally we study that the proposed node sleep strategy has significant energy saving compare with non-sleep cooperative transmission and direct transmission.

\subsection{Energy Modeling in Wireless Sensor Network}

The arrangement of cluster heads and other sensor nodes together in a wireless sensor network can be shown in figure 7 .

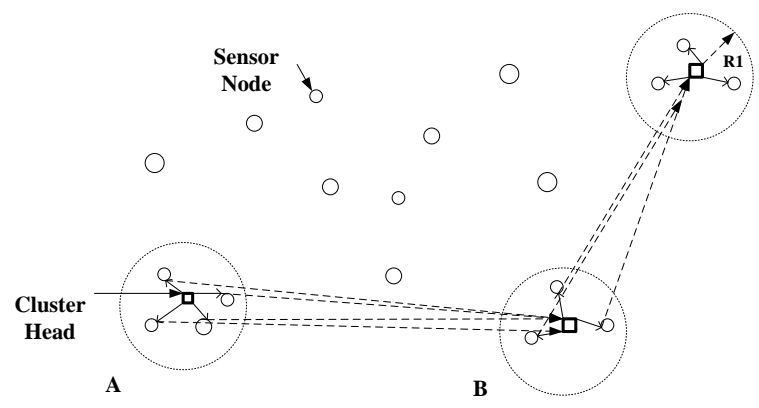

Fig.7: System model

Under this section description about the performance of intracluster broadcasting phase and inter-cluster cooperative transmission phase will be given.

\subsubsection{Intra-cluster Broadcasting phase}

During intra-cluster broadcasting phase, not all neighborhood nodes will correctly decode the broadcasting packet. As a result, we first derive the probability of errors and the average number of sensor nodes that correctly decode the packet during this intra-cluster broadcasting phase. We use packet error rate (PER) as the figure of merit for the detection of error. As shown in figure 7 the distance " $r$ " between $\mathrm{CH}$ and arbitrary neighboring node is considered as the random variable whose probability density function (PDF) is:

$$
f_{r}(r)=2 r / R_{1}^{2}, 0<r \leq R_{1} .
$$

For this transmission, we assume square law path loss with Rayleigh fading. So, according to link budget relationship [6] the received SNR $\left(\gamma_{1}\right)$

$$
\gamma_{1}=\frac{G E_{1}|h|^{2}}{r^{2} N_{o}}
$$

Where $\mathrm{h}$ is wireless channel gain between $\mathrm{CH}$ and sensor nodes in cluster and $|\mathrm{h}|$ follow Rayleigh distribution. No is the noise power E1 indicates the energy consumption per bit for transmission. For the given E1 and $r$, the average BER

Can be given by

$\mathrm{P}_{1}^{\mathrm{b}}=\mathrm{E}\left(\mathrm{Q}\left(\sqrt{2 \gamma_{1}}\right)\right) \approx \frac{\mathrm{r}^{2} \mathrm{~N}_{\mathrm{o}}}{4 \mathrm{GE}_{1}}$

Where, $\mathrm{Q}(\mathrm{x})$ is $\mathrm{Q}$ function. We set the packet length as $\mathrm{L}$ bits. The PER $\mathrm{P}_{1}^{\mathrm{p}}$ can be expressed as:

$\mathrm{P}_{1}^{\mathrm{p}}=1-\left(1-\mathrm{P}_{1}^{\mathrm{b}}\right)^{\mathrm{L}}$

(14)
And the average PER in cluster will be:

$\overline{\mathrm{P}_{1}^{\mathrm{P}}} \approx \int_{0}^{\mathrm{R}_{1}} \mathrm{~L}\left(\frac{\mathrm{r}^{2} \mathrm{~N}_{\mathrm{o}}}{4 \mathrm{GE}_{1}}\right) \frac{2 \mathrm{r}}{\mathrm{R}_{1}^{2}} \mathrm{dr}=\frac{\mathrm{LN}_{\mathrm{o}} \mathrm{R}_{1}^{2}}{8 \mathrm{GE}_{1}}$

Since the channels from the $\mathrm{CH}$ to the relay nodes are independent. Therefore, the number of nodes that successfully decode the packet is subjected to binomial distribution and probability that $\mathrm{m}$ nodes correctly decode the packet is:

$\mathrm{P}_{\mathrm{m}}(\mathrm{m})=\sum_{\mathrm{n}=\mathrm{m}}^{\infty}\left(\begin{array}{c}\mathrm{n} \\ \mathrm{m}\end{array}\right) \frac{\left(\rho \pi \mathrm{R}_{1}^{2}\right)^{\mathrm{n}} \mathrm{e}^{-\rho \pi \mathrm{R}_{1}^{2}}}{\mathrm{n} !}\left(\overline{\mathrm{P}_{1}^{\bar{p}}}\right)^{\mathrm{n}-\mathrm{m}}\left(1-\overline{\mathrm{P}_{1}^{\mathrm{P}}}\right)^{\mathrm{m}}$,

$\mathrm{m}=0,1,2 \ldots$. Then, the average number of nodes that correctly decoded packet in the cluster is

$\overline{\mathrm{m}}=\sum_{\mathrm{m}=0}^{\infty} \mathrm{m} \times \mathrm{P}_{\mathrm{m}}(\mathrm{m})=\rho \pi \mathrm{R}_{1}^{2}\left(1-\overline{\mathrm{P}_{1}^{\mathrm{p}}}\right)$

5.2.2 Inter-cluster Cooperative Transmission Phase As the distance between two clusters (A and B), shown in

Fig7 is usually much larger than cluster radius. The cooperative nodes transmit the packet using the DSTBC. We assume symbol level synchronization and perfect channel knowledge at the receiver.

Due to orthogonal property, the instantaneous received SNR of the CMISO system can be shown as:

$\gamma_{2}=\left(\frac{\mathrm{GE}_{2}}{\mathrm{~d}^{2} \mathrm{~N}_{\mathrm{o}}}\right)\|\mathrm{h}\|_{\mathrm{F}}^{2}$

Where $E_{2}$ shows transmission energy per bit per node and $d$ is transmission distance between two clusters, in which information is transmitting. So, average SNR at receiver side according to link budget relationship is:

$\overline{\gamma_{2}}=\mathrm{GE}_{2} /\left(\mathrm{d}^{2} \mathrm{~N}_{\mathrm{o}}\right)$

So, we get average BER of inter- cluster transmission.

$\mathrm{P}_{2}^{\mathrm{b}}=\int_{0}^{\infty} \mathrm{Q}\left(\overline{\gamma_{2}} \mathrm{x}\right) \mathrm{f}(\mathrm{x}) \mathrm{dx}$,

\subsubsection{Overall PER of the cooperative transmission phase}

The average overall PER " $\mathrm{P}$ all" of cooperative transmission is determined by the PER in both intra- and inter-cluster transmissions, which can be expressed as:

$$
\begin{aligned}
& \mathrm{P}_{\text {all }}^{\mathrm{p}}=\exp \left(\rho \pi \mathrm{R}_{1}^{2}\left(\overline{\mathrm{P}_{1}^{\mathrm{p}}}-1\right)\right) \\
& \times \sum_{\mathrm{m}=0}^{\infty}\left(\frac{\left(\rho \pi \mathrm{R}_{1}^{2}-\rho \pi \mathrm{R}_{1}^{2} \overline{\mathrm{P}_{1}^{\mathrm{p}}}\right)^{\mathrm{m}}}{\mathrm{m} !} \times \mathrm{P}_{2}^{\mathrm{p}}\right)
\end{aligned}
$$

\subsubsection{The overall energy consumption}

For the accurate calculation of energy consumption, both the transmission energy consumption and the associated circuit energy consumption are considered.

The overall energy consumption is consists of inter and intra cluster energy consumption. So it can give as:

$$
\mathrm{E}_{\text {intra }}=\mathrm{L}\left((1+\alpha) \mathrm{E}_{1}+\mathrm{E}_{\mathrm{ct}}+\rho \pi \mathrm{R}_{1}^{2} \mathrm{E}_{\mathrm{cr}}\right)
$$

Where $\alpha$ shows transmission efficiency of power amplifier

$$
\mathrm{E}_{\text {inter }}=\mathrm{L}\left((1+\alpha)(\overline{\mathrm{m}}+1) \mathrm{E}_{2}+(\overline{\mathrm{m}}+1) \mathrm{E}_{\mathrm{ct}}+\mathrm{E}_{\mathrm{cr}}\right.
$$

So, total energy consumption of each cooperative transmission is: 


$$
\begin{gathered}
\mathrm{E}_{\text {all }}=\mathrm{E}_{\text {intra }}+\mathrm{E}_{\text {inter }} \\
\mathrm{E}_{\text {all }}=\mathrm{L}\left((1+\alpha)\left(\mathrm{E}_{1}+\rho \pi \mathrm{R}_{1}^{2}\left(1-\overline{\mathrm{P}_{1}^{\mathrm{p}}}\right) \mathrm{E}_{2}\right)\right)+ \\
\mathrm{L}\left(\left(\rho \pi \mathrm{R}_{1}^{2}\left(1-\overline{\mathrm{P}_{1}^{\mathrm{p}}}\right)+2\right) \mathrm{E}_{\mathrm{ct}}+\left(\rho \pi \mathrm{R}_{1}^{2}+1\right) \mathrm{E}_{\mathrm{cr}}\right)
\end{gathered}
$$

Equation (24) represents that total energy consumption is affected by the following independent parameters: $L, E_{1}, E_{2}$, $\mathrm{R}_{1}$ etc.

\subsection{Performance comparison of nodes sleeps strategy with non-sleep cooperative transmission and direct transmission}

The performance comparison between transmission schemes given above will be shown for different terms as

- Nodes density

- Inter-cluster transmitting distance

- $\quad$ Cluster radius

- Intra-cluster energy consumption

For the system parameters (node active rate and energy consumption), the performance of the energy-optimized cooperative scheme is show under this topic for which different cases are discussed.

Case1: The density of sensor node vs. the optimal node active rate. In this case active rate $\eta$ is set as a variable. How optimal $\eta$ is affected by $\rho$ can be shown in the graphs [1]. When $\rho$ is smaller than a certain critical point, the optimal $\eta$ is equal to one. This is because in the cooperation, all the sensor nodes must participate to support the target overall PER. This indicates that number of sensor nodes should be higher in cluster to get higher $\mathrm{P}_{\text {all }}^{\mathrm{p}}$ to under the minimization of $\mathrm{E}_{\mathrm{all}}$.

Case2. Under this case there is a comparison of overall energy consumption of proposed scheme with those of non-sleep scheme $(\eta=1)$, relay transmission and direct transmission. In the relay transmission source node transmit the packet in the first time slot. In the second time slot, by using some criteria a relay node is selected from the cluster. In relay transmission scheme overall energy consumption can be given as:

$\mathrm{E}_{\text {relay }}=\mathrm{L}\left(\rho \pi \mathrm{R}_{1}^{2} \mathrm{E}_{\mathrm{cr}}+2 \mathrm{E}_{\mathrm{ct}}+2 \mathrm{E}_{\mathrm{t}, \mathrm{d}}\right)$

And in the direct communication overall energy consumption is given by:

$\mathrm{E}_{\text {direct }}=\mathrm{L}\left(\mathrm{E}_{\mathrm{cr}}+\mathrm{E}_{\mathrm{ct}}+\mathrm{E}_{\mathrm{t}, \mathrm{d}}\right)$

Where, $E_{t, d}$ is the transmitting energy consumption of the source. $E_{c r}$ is the receiving circuit energy consumption and $E_{c t}$ is the transmitting circuit energy consumption.

$\mu_{1}=\frac{E_{\text {all }}}{E_{\text {non }}}$

$\mu_{2}=\frac{E_{\text {all }}}{E_{\text {relay }}}$

$\mu_{3}=\frac{E_{\text {all }}}{E_{\text {direct }}}$

Literature says [1] when $\rho$ is small $\mu_{1}=1$. Thus two schemes have the same overall energy consumption. As $\rho$ increases, $\mu_{1}$ decreases greatly. This indicates that sleep strategy plays an important role in saving energy and when $\rho$ is higher it becomes more efficient.

Case3: The inter-cluster distance vs. overall energy consumption. In this case $\mu_{1}, \mu_{2}, \mu_{3}$ are introduced to discuss how the inter-cluster distance affects the overall energy consumption. Here the value of is set as $\rho=0.05$ and other parameters are same. According to some surveys, when $d$ increases, $\mu_{1}$ increases correspondingly. This is because our scheme needs more sensor nodes in activation which take part in cooperation when $d$ is increases. Therefore, this scheme increases $\eta$ and then overall energy consumption reach nearer to the non-sleep cooperative scheme [1].

Case4: cluster radius vs. the minimized overall energy consumption and the optimal sensor node active rate. In this case it shows how both energy consumption and node active rate is varied according to cluster radius $R$ [1]. This investigation shows that $E_{\text {all }}$ exist at its minimum value when $\mathrm{R}$ increases and when $\mathrm{R}$ is small, cooperative sensor nodes in the cluster is quite small and hence need higher energy to reach the target. Cluster radius affects the optimal active rate as when cluster radius is smaller than a certain critical point $(\eta=1)$ which corresponds to the situation that there are few cooperative sensor nodes in the cluster and when cluster radius is larger than no. of sensor nodes start falling in cluster becoming larger.

Case 5: Intra-cluster broadcasting energy consumption vs. the minimized overall energy consumption. This case is interesting as it shows that minimization of $E_{a l l}$ does not always decrease with $E_{1}$ and even it exists as an optimal value of $E_{1}$. It can be clear as: there will be more no. of nodes that can correctly decode the packet and can participate in the cooperative transmission when $\mathrm{E}_{1}$ is increases and during cooperative transmission it also introduces additional transmitting energy $\left(\mathrm{E}_{\mathrm{ct}}\right)$. When there is a decrease in the value of $E_{1}$ the additional inter-cluster transmitting energy consumption to reach target PER must be increased by the network. This kind of simulation suggests us that jointly the performance of the network can be optimized by more parameters.

\section{OTHER TYPES OF WIRELESS SENSOR NETWORKS}

Other than random wireless sensor network, there are other famous networks which are generally used in wireless field. Out of them one is wireless Ad-hoc network (WANET) which is a decentralized type of wireless network and it is called as Ad-hoc because it does not rely on a pre-existing infrastructure such as routers in wired network process point [16].

Recently CMIMO technique and its variations like CMISO, are adopted by ad-hoc networks in wireless sensor network for the improvement in energy efficiency over SISO systems by exploiting the spatial diversity enhanced from cooperative communication [14]-[15]. These ad-hoc networks are also of great use in requirements of major applications.

\section{CONCLUSION}

In this paper WSN and WSN nodes are explained briefly and had a brief idea about the energy saving approaches in WSN which is quite important for research in this subject. It has been shown that how the MIMO systems are more reliable and energy efficient than the SISO systems by describing its energy model. But it may be mislead when both transmission energy and circuit energy are considered, because of the complex circuitry of the MIMO system. Then CMIMO has been 
introduced which compensates this problem and the main properties of CMIMO are also mentioned in the paper and gave its comparison with DCA. CMIMO energy model for cluster system is also described in the paper and then there is an introduction about the node sleep strategy in CMIMO and then consider that how the various system constraints affect the optimization.

\section{REFERENCES}

[1] Bin Li, Hongxiang, Wenjie Wang, Quiye Yin and Hui Liu "Performance analysis and optimization for energyefficient cooperative transmission in random wireless sensor network," IEEE trans wireless comm. Vol.12, pp. 4647-4656, September 2013.

[2] Vibhav kr. Sachan, Syed Akhtar Imam, M.T. Beg “ Energy efficient communication methods in wireless sensor networks: a critical review", international journal of computer applications, Vol. 39, pp.35-48, February 2012

[3] W. Heinzelman, A. Chandrakasn, and H. Balarislman, "An application specific protocol architecture for wireless microsensor networks, "IEEE Trans. Wireless commun., vol.1, pp. 660-670, October 2002.

[4] M. Z. Siam, M. Krunz, and O. Younis, "Energy efficient clustering/routing for cooperative MIMO operation in sensor networks," in proc. 2009 IEEE INFOCOM, PP. 621-629.

[5] A. Paulraj, R. Nabar, and D. Gore, "Introduction to SpaceTime wireless communication systems", Cambridge, U.K.: Cambridge university. Press, 2003, preprint.

[6] S. K. Jayaweera, "Virtual MIMO based co-operative communication for energy constrained wireless sensor networks," IEEE Trans wireless commun., vol.5., pp. 984-989, May 2006.

[7] D. Wu. Y. Cai, L Zhou, and J. Wang, "A cooperative communication scheme based on coalition formation game in clustered wireless sensor networks", IEEE Trans. Wireless commun. Vol.11, pp. 1190-1200, March 2012.

[8] S. Almouti, "A simple transmit diversity technique for wireless communications," IEEE J. Select. Areas Commun., vol.16, pp.1451-1458, oct.1998.
[9] T. H. Lee, "The design of CMOS Radio- Frequency Integrated Circuits, Cambridge, U.K.: Cambridge Univ.press, 1998.

[10] M. Steyaert, B.De, P. Leroux, M.Borremans, and K. Mertens, "Low- voltage low-power CMOS RF transceiver design" IEEE Trans. Microwave theory Tech., vol.50, PP.281-287, January 2002.

[11] S. Willingham, M.Perrott, B.Setterberg, A. Grzegorek, and B. McFarland, "An integrated $2.5 \mathrm{GHz} \Sigma \Delta$ frequency synthesizer with $5 \mu \mathrm{sec}$ and $2 \mathrm{Mb} / \mathrm{sec}$ closed loop modulation," in proc. ISSCC 2000, 2000, pp.138-139.

[12] P.J. Sullivan, B.A. Xavier, and W.H. Ku, "Low voltage performance of a microwave CMOS Gilbert cell mixer," IEEE J. Solid-State Circuits. Vol.32, pp.1151-1155, July 1997.

[13] S. Boyd and L. Vandenberghe, "Convex optimization" Cambridge, U.K.: Cambridge Univ. press, 2003.

[14] S. Cui, A. J. Goldsmith, and A. Bahai, "Energy Efficiency of MIMO and CMIMO techniques in sensor network". IEEE J. Sel. Areas Commun, vol.22, pp. 1089-1098, August 2004.

[15] Jun Zhang, Li Fei, Qiang Gao, Xiao-Hong peng "Energy Efficient Multi-hop cooperative MISO transmissiom with optimal Hop distance in Wireless Ad Hoc networks", IEEE Trans wireless commun. Vol.10, October 2011.

[16] Vibhav Kumar Sachan, Syed Akhtar Imam and M.R. Khan, "Integrated Energy Analysis of Space Time Block Codes for Energy Efficient RF Sensor Networks" International Journal of Telecommunication and Radio Engineering, Russia, Vol.71, Issue 19, PP. 1769-1780, Dec. 2012.

[17] Vibhav Kumar Sachan, Syed Akhtar Imam "Performance Analysis of Cooperative MIMO Techniques in Wireless Sensor Networks". The IUP Journal of Telecommunication, INDIA, Vol.4, No. 2, PP. 32-41, May 2012

[18] Vibhav Kumar Sachan, Ankur Gupta, Avinash Kumar; "Performance Analysis of MIMO Space Diversity Technique for Wireless Communications," Journal of Active and Passive Electronics Devices, USA, Vol.7, No. 1-2, pp. 51-59, Jan' 2012. 